November 1, 2018

\title{
Transition to Chaos in Discrete Nonlinear Schrödinger Equation with Long-Range Interaction
}

\author{
Nickolay Korabel ${ }^{a, 1}$, George M. Zaslavsky ${ }^{a, b}$ \\ a Courant Institute of Mathematical Sciences, New York University \\ 251 Mercer Street, New York, NY 10012, USA, \\ ${ }^{b}$ Department of Physics, New York University, \\ 2-4 Washington Place, New York, NY 10003, USA
}

\begin{abstract}
Discrete nonlinear Schrödinger equation (DNLS) describes a chain of oscillators with nearest neighbor interactions and a specific nonlinear term. We consider its modification with long-range interaction through a potential proportional to $1 / l^{1+\alpha}$ with fractional $\alpha<2$ and $l$ as a distance between oscillators. This model is called $\alpha$ DNLS. It exhibits competition between the nonlinearity and a level of correlation between interacting far-distanced oscillators, that is defined by the value of $\alpha$. We consider transition to chaos in this system as a function of $\alpha$ and nonlinearity. It is shown that decreasing of $\alpha$ with respect to nonlinearity stabilize the system. Connection of the model to the fractional genezalization of the NLS (called FNLS) in the long-wave approximation is also discussed and some of the results obtained for $\alpha$ DNLS can be correspondingly extended to the FNLS.
\end{abstract}

PACS: 45.05.+x; 45.50.-j; 45.10.Hj

Keywords: Long-range interaction, Discrete NLS, Fractional equations, Spatio-temporal chaos

\section{Introduction}

Nonlinear Schrödinger equation (NLS) is a paradigmatic equation that describes a slowly varying enveloping process in the nonlinear dispersive media. Applications of the NLS has been found in almost all important areas of physics: nonlinear optics, plasma physics, hydrodynamics, condenced matter physics, biology and others. The literature on NLS is extensive and the reviews [1, 2, 3, 4, 5, 6, can provide a strong impression on the importance of the subject. The NLS per se is an integrable system while its different types of perturbations, more related to the practical needs, include a broad spectrum of solutions from solitons and breathers to chaos and spatio-temporal turbulence.

\footnotetext{
${ }^{1}$ Corresponding author. Tel.: +1 212998 3260; fax: +1 2129954640.

E-mail address: korabel@cims.nyu.edu.
} 
Between different types of perturbations one can single out two the most interesting classes: external time-space dependent perturbation, and discretization of the NLS presented by a kind of space-difference equation, called discrete NLS (DNLS). It was shown in a set of publications (see [7, 8, 9] and references therein) that specific hyperbolic structure of the NLS phase space leads to the transition from a soliton type dynamics to different kind of chaos, including spatio-temporal turbulence, under even small perturbation. The fact that discretization induces chaos is fairly well known and, particularly, for the DNLS it was studied in [10, 11, 12, 13, 14] and [15, 16]. Destruction of solitons, breathers, and wave trains is similar to what occurs from external perturbations. A general physical mechanism of the onset of chaos induced by a discretization is known: transition from a differential equation to the difference one is equivalent to the appearance of a high frequency (in time or in space) periodic perturbation [17.

One can also consider DNLS as a separate problem that describes a chain of coupled manyparticles (oscillators) with local or nonlocal interaction. Such a system presents a specific interest for studying transition to chaos, turbulence, and statistical equilibrium in many-body problem. A new attraction at that point is the long-range interaction (LRI) that was introduced in an exponential Kac-Baker form in [18, 19] and later in a power Lennard-Jones form in [20]. In another version of the latter case the interaction between oscillators located at the positions $(n, m), n \neq m$, is proportional to $1 /|n-m|^{1+\alpha}$. The corresponding DNLS will be called $\alpha$ DNLS. Studying of such systems has multiple interest: transition to chaos and turbulence in the presence of LRI [21, 22], sinchronization in systems with many particles 23], controling chaos [24], and different manipulations with physical objects in optics [1, 25] and condenced matter [26, 27. All these physical features, being described by $\alpha$ DNLS, are functions of $\alpha$.

For the number of particles $N=4$ the DNLS equation can be solved exactly. For $N>4$ the DNLS is not integrable and chaotic solutions are possible. It was shown in 13, 15, 28, 29, 30, 31] that there are two main mechanisms responsible for chaotization of the DNLS. One of the mechanisms prevails for symmetric initial conditions and another for asymmetric ones. For symmetric initial conditions chaos was shown to emerge from the proximity to homoclinic orbits. For asymmetric initial conditions this mechanims do not play significant role, instead, perturbations induced by discretization and round-off errors cause random flipping of wave's direction of motion.

The primary goal of this paper is to study the transition to chaos in $\alpha$ DNLS depending on a parameter $0<\alpha<2$ that is responsible for the appearance of a power-like tail in solution, i.e. for the delocalization of modes. Parameter $\alpha$ has a simple physical meaning: it describes a level of collective coupling of particles. Particularly, for $\alpha=\infty$ we have only nearest neighbor interaction and for $\alpha=-1$ we have mean field type model.

Recenly, different properties of the nonlocal DNLS were studied in [32, 33, 34, 35. It was found that some properties of solutions depend on the interaction exponent $\alpha$ in the LRI case or on the exponent $\beta$ for the Kac-Baker interactions. Namely, for the power-law interaction it was shown that for $\alpha$ less than some critical value $\alpha_{c r}$, there is an interval of bistability where three posible stationary states exist at each value of some excitation number $M$ that characterize a level of nonlinearity. The first type of solution is a continuum-like mode, the second is an intrinsically localized breathing state and the third one is stationary itermediate state. The long-distance behavior of the intrinsically localized states depends on $\alpha$. For $\alpha>2$ their tails are exponential, while for $0<\alpha<2$ the tails are algebraic. By changing an interaction constant $J$, a stable solution may become unstable. A small symmetric force applied to the system can also trigger transigions from one type of stable solutions to another [33]. The continuum limit 
of this model was shown to be a nonlocal NLS equation [32. Another version of the nonlocal (integro-differential) NLS equation was proposed in [36]. Unlike the usual NLS equation, this nonlocal NLS equation has stationary solutions only in a finite interval of excitation numbers $\left[0, M_{\max }\right]$.

In a similar way, as dynamics of a chain of coupled oscillators can be reduced to the wave equation in the long wave-length limit $k \rightarrow 0$, the chain of $\alpha$ DNLS can be reduced to the fractional generalization of NLS equation or Ginzburg-Landau equation (FGL) [37, 38, 39, 23. 40, 41. It was shown in 39, 23, that mapping of the $\alpha$ DNLS equation to fractional NLS (FNLS), or similarly to FGL, can be realized by some transform operator. In all fractional equations of this type second coordinate derivative is replaced by the fractional Riesz derivative [42] of order $\alpha$. The corresponding comparison of solutions of discrete chain of oscillators with the sinepotential and fractional generalization of the sine-Gordon equation was perfomed in [27, 43]. The latter results of [43] confirm a path to the dual features of the systems with LRI of order $\alpha$ and the systems described by a corresponding equation with fractional derivative [39, 23.

In this paper we provide a detailed study of the transition to chaos in $\alpha$ DNLS depending on the parameter $0<\alpha<2$. It is well known that fractional values of $\alpha$ appears in numerous complex systems such as spin-interacting systems [44, adatoms [26], colloids [45], chemical surfaces [46], quantum field theory [47, 48, 49], etc. In correspondence to the results [39, 23, 40], the obtained properties of the $\alpha$ DNLS can be extended to the FNLS. It will be shown that onset of chaos follows as a result of competition between the nonlinearity level and the level of coherency of the chain of oscillators defined by the value of $\alpha$.

\section{Basic equations}

The continuous Nonlinear Schrödinger (NLS) equation defined on the finite interval $[-L / 2, L / 2]$ with the periodic boundary conditions $\psi(x+L, t)=\psi(x, t)$ can be written as

$$
i \frac{d \psi}{d t}+\gamma|\psi|^{2} \psi+\frac{\partial^{2} \psi}{\partial x^{2}}=0
$$

where $\gamma$ is a constant ( $\gamma=1$ corresponds to the focusing nonlinearity). In turn, the discrete nonlinear Schrödinger equation (DNLS) which describes a lattice of $N$ anharmonic oscillators with nearest-neighbors interaction is defined as

$$
i \frac{d \psi_{n}}{d t}+\gamma\left|\psi_{n}\right|^{2} \psi_{n}+\epsilon\left(\psi_{n+1}-2 \psi_{n}+\psi_{n-1}\right)=0, \quad(n=1, \ldots, N),
$$

where $\psi_{n+N}=\psi_{n}, \forall n$ is the periodic boundary condition. The quantity $\psi_{n}=\psi_{n}(t)$ is the complex amplitude of the oscillator at site $n$. With $\epsilon=1 /(\Delta x)^{2}$, Eq. (2) is seen as a standard finite difference approximation to Eq. (1). Here $\Delta x=L / N$ is a distance between oscillators.

The Hamiltonian, $H$, and the excitation number (or norm), $M$,

$$
H=\sum_{n=1}^{N}\left(\epsilon\left|\psi_{n+1}-\psi_{n}\right|^{2}-\frac{\gamma}{2}\left|\psi_{n}\right|^{4}\right), \quad M=\sum_{n=1}^{N}\left|\psi_{n}\right|^{2},
$$

are the conserved quantities. 
The model which we study in the following is described by the Hamiltonian

$$
H=\frac{1}{2} \sum_{n=1}^{N}\left(\sum_{\substack{m=1 \\ m \neq n}}^{N} J_{n-m}\left|\psi_{m}-\psi_{n}\right|^{2}-\gamma\left|\psi_{n}\right|^{4}\right)
$$

with the periodic boundary conditions: $\psi_{n+N}=\psi_{n}$. The coupling function is defined by

$$
J_{n-m}=\frac{J}{|n-m|^{1+\alpha}}, \quad(n \neq m),
$$

where $J$ is a coupling constant and $\alpha$ is an exponent which, depending on the physical situation, can be integer or fractional. The standard nearest-neighbor DNLS equation is recovered in the limit $\alpha \rightarrow \infty$. From the Hamiltonian Eq. (44) one obtain the equation of motion

$$
\begin{gathered}
i \frac{d \psi_{n}}{d t}=\partial H / \partial \psi_{n}^{*}, \\
i \frac{d \psi_{n}}{d t}+\gamma\left|\psi_{n}\right|^{2} \psi_{n}+\sum_{\substack{m=1 \\
m \neq n}}^{N} J_{n-m}\left(\psi_{n}-\psi_{m}\right)=0, \quad(n=1, \ldots, N) .
\end{gathered}
$$

Consider the infinite chain of equidistant oscillators $(N \rightarrow \infty$ in Eq. (7) $)$. The Fourier transform of $\psi_{n}$ is given as

$$
\hat{\psi}_{n}(k, t)=\sum_{n=-\infty}^{\infty} \psi_{n}(t) \exp \left(-i k x_{n}\right) \equiv \mathcal{F}_{\Delta}\left\{\psi_{n}(t)\right\},
$$

where $x_{n}=n \Delta x$ is a coordinate of the $n$-th oscillator, and $\Delta x=2 \pi / K$ is a distance between oscillators. Here we have treated $k$ as a continuous variable. The inverse transform is defined as

$$
\psi_{n}(t)=\frac{1}{K} \int_{-K / 2}^{+K / 2} d k \hat{\psi}_{n}(k, t) \exp \left(i k x_{n}\right) \equiv \mathcal{F}_{\Delta}^{-1}\left\{\hat{\psi}_{n}(t)\right\},
$$

Transition to the continuous limit $\Delta x \rightarrow 0(K \rightarrow \infty)$ can be obtained by transforming $\psi_{n}(t)=$ $\psi(n \Delta x, t)=\psi\left(x_{n}, t\right) \rightarrow \psi(x, t)$. Then, changing sums into integrals, Eqs. (87), (9) become

$$
\begin{gathered}
\tilde{\psi}(k, t)=\int_{-\infty}^{+\infty} d x e^{-i k x} \psi(x, t) \equiv \mathcal{F}\{\psi(x, t)\}, \\
\psi(x, t)=\frac{1}{2 \pi} \int_{-\infty}^{+\infty} d k e^{i k x} \tilde{\psi}(k, t) \equiv \mathcal{F}^{-1}\{\tilde{\psi}(k, t)\},
\end{gathered}
$$

where

$$
\tilde{\psi}(k, t)=\mathcal{L} \hat{\psi}(k, t), \quad \psi(x, t)=\mathcal{L} \psi_{n}(t)=\mathcal{L} \psi\left(x_{n}, t\right),
$$

and $\mathcal{L}$ denotes the limit $\Delta x \rightarrow 0$. Operation $\bar{T}=\mathcal{F}^{-1} \mathcal{L} \mathcal{F}_{\Delta}$ can be called a transform operator (or transform map), since it performs a tranfrormation of a discrete model of coupled oscillators to the continuous media. For $0<\alpha<2$ application of $\bar{T}$ leads to the fractional NLS. For more 
details, definitions and applications of the transform operator to different systems see [23, 40, 43]. In a brief form this approximation is as follows [39, 23].

First we perform the Fourier tranformation of Eq. (17) for infinite chain of oscillators $(N \rightarrow \infty)$

$$
i \frac{\partial \hat{\psi}(k, t)}{\partial t}+\gamma \mathcal{F}_{\Delta}\left\{\left|\psi_{n}\right|^{2} \psi_{n}\right\}+J\left(\hat{J}_{\alpha}(k)-\hat{J}_{\alpha}(0)\right) \hat{\psi}(k, t)=0
$$

where

$$
\hat{J}_{\alpha}(k)=\sum_{\substack{n=-\infty \\ n \neq 0}}^{+\infty} \frac{e^{-i k n \Delta x}}{|n|^{1+\alpha}}=\sum_{n=1}^{+\infty} \frac{e^{-i k n \Delta x}+e^{i k n \Delta x}}{n^{1+\alpha}}=L i_{1+\alpha}\left(e^{i k \Delta x}\right)+L i_{1+\alpha}\left(e^{-i k \Delta x}\right),
$$

and $L i_{1+\alpha}(z)$ is a polylogarithm function [50] with series representation

$$
\hat{J}_{\alpha}(k)=a_{\alpha}|\Delta x|^{\alpha}|k|^{\alpha}+2 \sum_{n=0}^{\infty} \frac{\zeta(1+\alpha-2 n)}{(2 n) !}(\Delta x)^{2 n}\left(-k^{2}\right)^{n}, \quad|k|<1, \quad \alpha \neq 0,1,2,3 \ldots
$$

Here $J_{\alpha}(0)=2 \zeta(1+\alpha), \zeta$ is the Riemann zeta-function and

$$
a_{\alpha}=2 \Gamma(-\alpha) \cos \left(\frac{\pi \alpha}{2}\right) .
$$

For $\alpha=2, \hat{J}_{\alpha}(k)$ reduces to the Clausen function $C l_{2}(k)$ [51].

Substitution of Eqs. (15) and (16) into (13) gives

$$
\begin{gathered}
i \frac{\partial \hat{\psi}(k, t)}{\partial t}+J \hat{\psi}(k, t)\left(a_{\alpha}|\Delta x|^{\alpha}|k|^{\alpha}+2 \sum_{n=0}^{\infty} \frac{\zeta(\alpha+1-2 n)}{(2 n) !}(\Delta x)^{2 n}\left(-k^{2}\right)^{n}-\hat{J}_{\alpha}(0)\right)+ \\
+\gamma \mathcal{F}_{\Delta}\left\{\left|\psi_{n}\right|^{2} \psi_{n}\right\}=0 .
\end{gathered}
$$

Note that $\hat{J}_{\alpha}(0)$ exactly cancels the constant which is the first term of the sum in Eq. (17). In the limit $k \rightarrow 0$ Eq. (17) yields

$$
i \frac{\partial \hat{\psi}(k, t)}{\partial t}+\bar{J} \hat{\mathcal{T}}_{\alpha, \Delta}(k) \hat{\psi}(k, t)+\gamma \mathcal{F}_{\Delta}\left\{\left|\psi_{n}\right|^{2} \psi_{n}\right\}=0,
$$

where $\bar{J}=J|\Delta x|^{\min \{\alpha, 2\}}$ and

$$
\hat{\mathcal{T}}_{\alpha, \Delta}(k)=\left\{\begin{array}{lll}
a_{\alpha}|k|^{\alpha}-|\Delta x|^{2-\alpha} \zeta(\alpha-1) k^{2}, & 0<\alpha<2, & (\alpha \neq 1) \\
|\Delta x|^{\alpha-2} a_{\alpha}|k|^{\alpha}-\zeta(\alpha-1) k^{2}, & 2<\alpha<4, & (\alpha \neq 3) .
\end{array}\right.
$$

For $0<\alpha<2$ the operator $\hat{\mathcal{T}}_{\alpha, \Delta}(k)$ is defined up to $O\left(k^{2}\right)$ and for $2<\alpha<4$ up to $O\left(|k|^{\alpha}\right)$.

Eq. (19) has a crossover scale for

$$
k_{0}=\left|a_{\alpha} / \zeta(\alpha-1)\right|^{1 /(2-\alpha)}|\Delta x|^{-1} .
$$

From Eq. (20) it follows that $\hat{\mathcal{T}}_{\alpha, \Delta}(k) \sim k^{2}$ for $\alpha>2, k \ll k_{0}$ and nontrivial expression $\hat{\mathcal{T}}_{\alpha, \Delta}(k) \sim|k|^{\alpha}$ appears only for $\alpha<2, k \ll k_{0}$. This crossover was considered also in [39, 23]. 
Performing the transition to the limit $k \ll k_{0}$ (or more precisely $k \Delta x \ll k_{0} \Delta x$ ) and applying the inverse Fourier transform to (18), we obtain

$$
i \frac{\partial}{\partial t} \psi(x, t)+\bar{J} \mathcal{T}_{\alpha}(x) \psi(x, t)+\gamma|\psi(x, t)|^{2} \psi(x, t)=0 \quad \alpha \neq 0,1,2, \ldots
$$

where

$$
\begin{gathered}
\mathcal{T}_{\alpha}(x)=\mathcal{F}^{-1}\left\{\hat{\mathcal{T}}_{\alpha}(k)\right\}= \begin{cases}-a_{\alpha} \frac{\partial^{\alpha}}{\partial|x|^{\alpha}}, & 0<\alpha<2, \quad(\alpha \neq 1) \\
\zeta(\alpha-1) \frac{\partial^{2}}{\partial x^{2}}, & \alpha>2, \quad(\alpha \neq 2,3,4, \ldots) ;\end{cases} \\
\hat{\mathcal{T}}_{\alpha}(k)=\left\{\begin{array}{ll}
a_{\alpha}|k|^{\alpha}, & 0<\alpha<2, \quad(\alpha \neq 1) \\
-\zeta(\alpha-1) k^{2}, & \alpha>2,
\end{array} \quad(\alpha \neq 2,3,4, \ldots) .\right.
\end{gathered}
$$

Here, we have used the connection between the Riesz fractional derivative and its Fourier transform [42]:

$$
|k|^{\alpha} \longleftrightarrow-\frac{\partial^{\alpha}}{\partial|x|^{\alpha}}, \quad k^{2} \longleftrightarrow-\frac{\partial^{2}}{\partial x^{2}}
$$

Properties of the Riesz fractioanl derivative can be found in [42, 52, 53, 54].

In the following section we will consider simulations of the finite chain of oscillators on a finite interval $(-L / 2, L / 2)(n=1, \ldots, N$, where $N$ is even) described by the Hamiltonian

$$
H=\sum_{n=1}^{N}\left(J \sum_{l=1}^{N / 2-1} \frac{\left|\psi_{n+l}-\psi_{n}\right|^{2}}{l^{1+\alpha}}-\frac{\gamma}{2}\left|\psi_{n}\right|^{4}\right)
$$

and equation of motion

$$
i \frac{d \psi_{n}}{d t}+\gamma\left|\psi_{n}\right|^{2} \psi_{n}+J \sum_{l=1}^{N / 2-1} \frac{\psi_{n+l}-2 \psi_{n}+\psi_{n-l}}{l^{1+\alpha}}=0, \quad(n=1, \ldots, N) .
$$

The form of the Hamiltonian and the equation of motion account for the periodic boundary conditions $\psi_{n+N}=\psi_{n}$. To avoid double counting of interactions we introduce a cutoff for the interaction range at $N / 2-1$.

All initial conditions and parameters are choosen to make it possible to use the transform operator $\bar{T}$. This means that for not too large time the obtained results can be also applied to the FDNLS equation (21)

$$
\begin{gathered}
i \frac{\partial}{\partial t} \psi(x, t)-G \frac{\partial^{\alpha} \psi(x, t)}{\partial|x|^{\alpha}} \psi(x, t)+\gamma|\psi(x, t)|^{2} \psi(x, t)=0, \quad 0<\alpha<2, \quad \alpha \neq 1, \\
G=2 J|\Delta x|^{\alpha} \Gamma(-\alpha) \cos (\pi \alpha / 2) .
\end{gathered}
$$

It is also worthwile to mention that for $\alpha \rightarrow 2$ we satisfy the conditions of the continuous limit approach since $k_{0} \rightarrow \infty$, that means the corresponding results should be close to the solution of NLS. Thus, considering $\alpha$ far from $\alpha=2$, one can compare the solutions of $\alpha$ DNLS with the solutions of DNLS with the nearest neighbor interaction. Such results provide a role of the long-range interaction for the chain dynamics. 


\section{Numerical results}

In this section numerical results obtained from solutions of the equations of motion Eq. (25) on the finite interval $(-L / 2, L / 2)$ are summarized. Parameter $J$ was normalized to $J / J_{0}$, where

$$
J_{0}=\sum_{n=1}^{N / 2-1} \frac{1}{|n|^{1+\alpha}} .
$$

For all sets of parameters we integrate the equations of motion Eq. (25) up to the time $T=100$. Numerical solutions were stored at each $t_{q}=q T / Q, q=0, . ., Q-1$, where $Q=10^{3}$. The number of oscillators was $N=32$ in all simulations.

To visualise the numerical solution we plot the surface $|\psi(x, t)|^{2}$ and the "phase portrait" of the central oscillator $\left(n=0, x_{n}=0\right)$ formed by the variables:

$$
A(t)=|\psi(0, t)|^{2}, \quad A_{t}=d A / d t .
$$

Also we plot $\operatorname{Im}(\psi(0, t))$ vs. $\operatorname{Re}(\psi(0, t))$ and the phase difference of two nearby trajectories $\psi(0, t), \psi(\Delta x, t)$

$$
d f=\tan ^{-1}(\operatorname{Re}(\psi(0, t)) / \operatorname{Im}(\psi(0, t)))-\tan ^{-1}(\operatorname{Re}(\psi(\Delta x, t)) / \operatorname{Im}(\psi(\Delta x, t))),
$$

where $\Delta x=L / N$. We also calculate the discrete Fourier transform of the sequence $\psi\left(0, t_{q}\right)$, which is defined

$$
\begin{gathered}
\hat{\psi}_{n}\left(w_{j}\right)=\frac{1}{Q} \sum_{q=0}^{Q-1} \psi_{n}\left(t_{q}\right) \exp \left(-i w_{j} t_{q}\right), \\
\psi_{n}\left(t_{q}\right)=\sum_{j=0}^{Q-1} \hat{\psi}_{n}\left(w_{j}\right) \exp \left(i w_{j} t_{n}\right),
\end{gathered}
$$

where the wavenumber is $w_{j}=2 \pi j / Q, j=0, \ldots, Q-1$. The corresponding power spectrum $S$ of the sequence $\psi_{n}\left(t_{q}\right)(q=0, \ldots, Q-1)$ is given by

$$
S_{j} \equiv S\left(w_{j}\right)=\left|\hat{\psi}_{n}\left(w_{j}\right)\right|^{2} .
$$

In our simulation we use two types of initial conditions: symmetric and asymmetric types. Symmetric initial conditions are defined as

$$
\psi(x, 0)=a+b \cos \left(\frac{2 \pi}{L} x\right)
$$

with constants $a=0.5, b=0.1$ and $L=2 \sqrt{2} \pi$. This form of symmetric initial conditions was also used in [15, 28] to study chaos in discretizations of standard NLS equation. For asymmetric initial conditions we use the following expression

$$
\psi(x, 0)=a\left[1+b\left\{e^{i c} \cos \left(\frac{2 \pi}{L} x\right)+e^{i d} \sin \left(\frac{2 \pi}{L} x\right)\right\}\right],
$$

where $a=1, b=0.2, c=0.9, d=2.03$ and $L=2 \sqrt{2} \pi$. The form of the initial condition is taken the same as in [13]. 

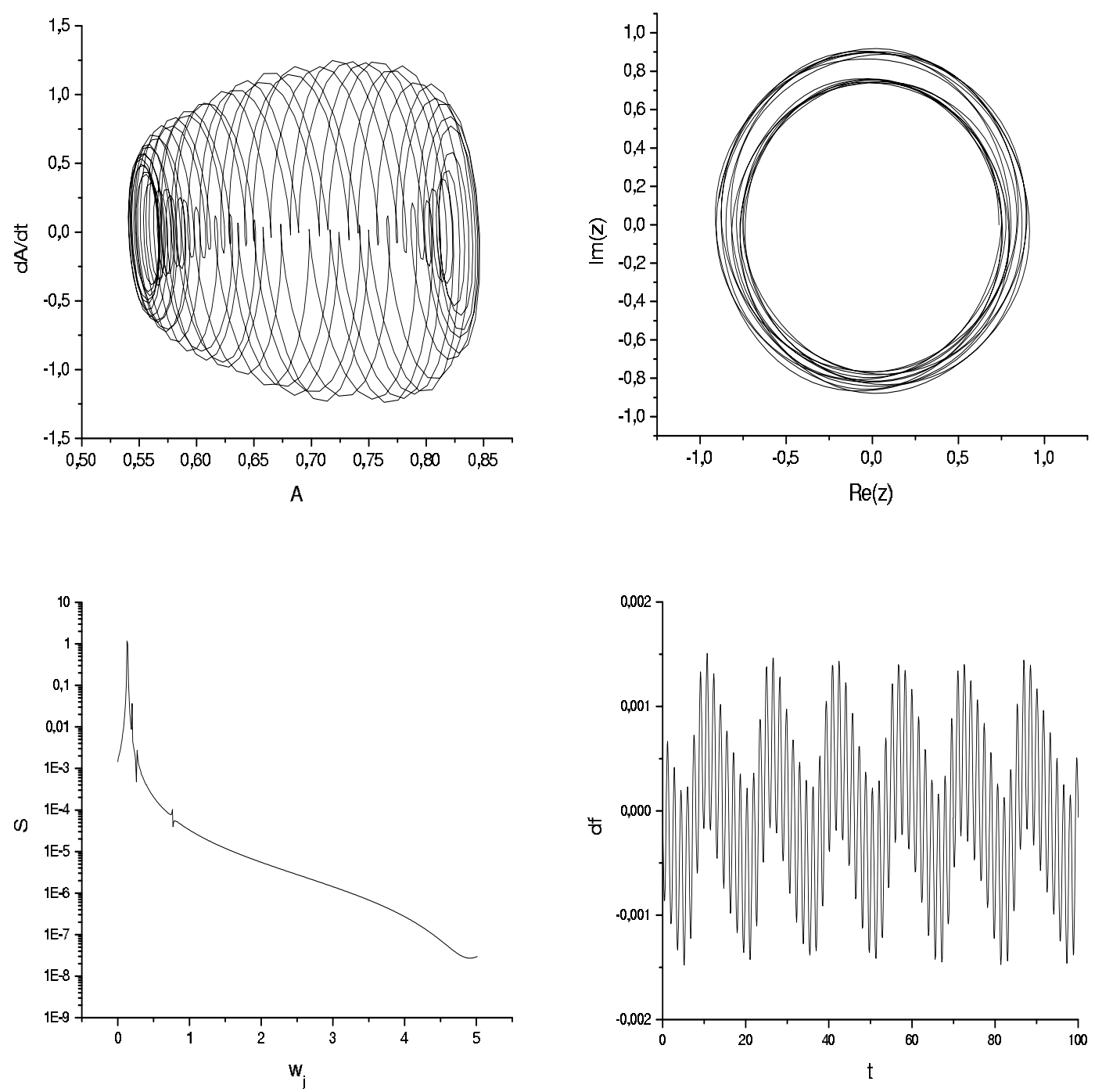

Figure 1: Time evolution of the central oscillator. The values of parameters are $\alpha=1.11$, $J / J_{0}=0.7$ and $M=12.5$. The initial condition is given by Eq. (34). 

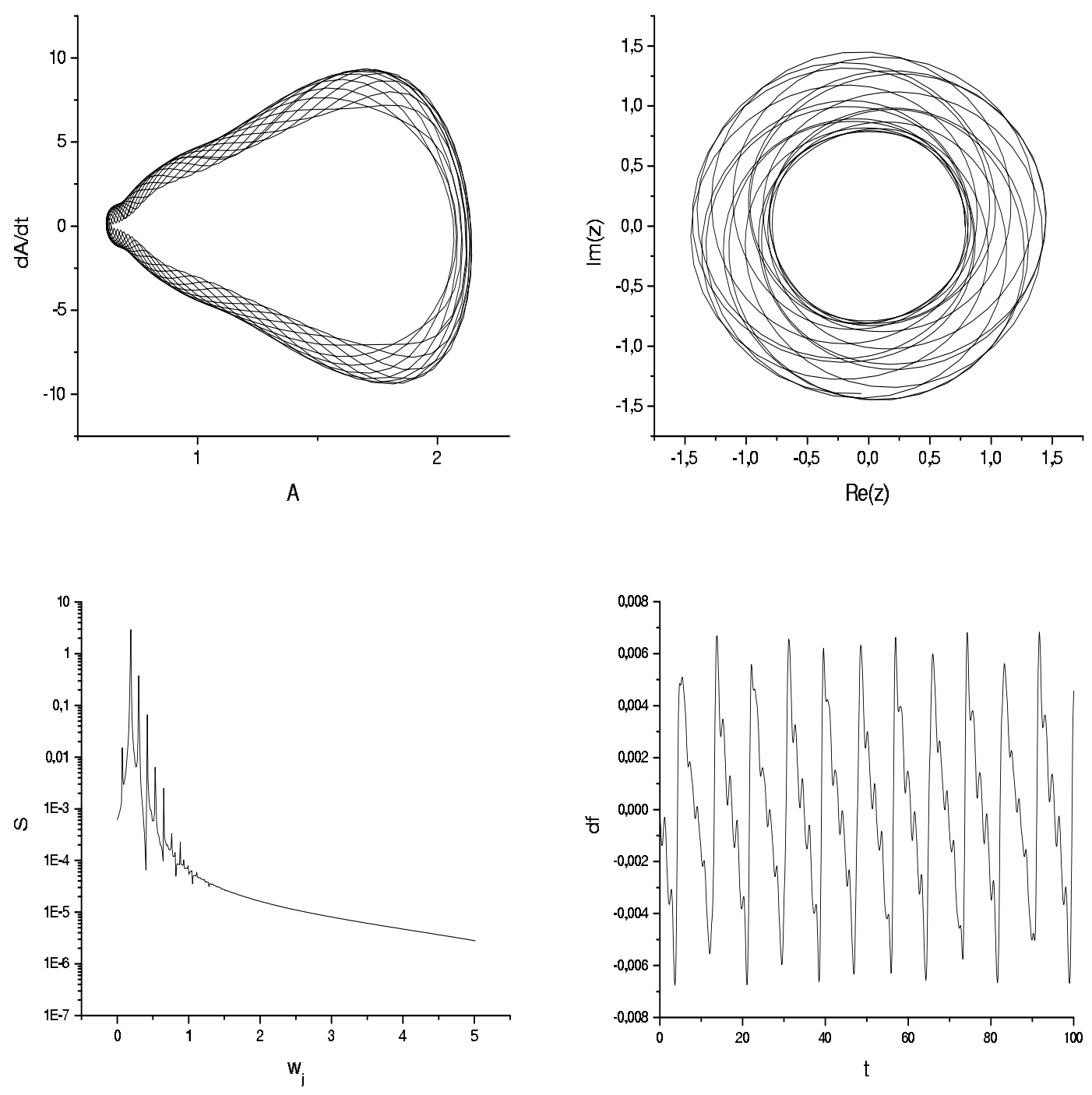

Figure 2: Time evolution of the central oscillator. The values of parameters are $\alpha=1.11$, $J / J_{0}=0.7$ and $M=14.28$. The initial condition is given by Eq. (34). 

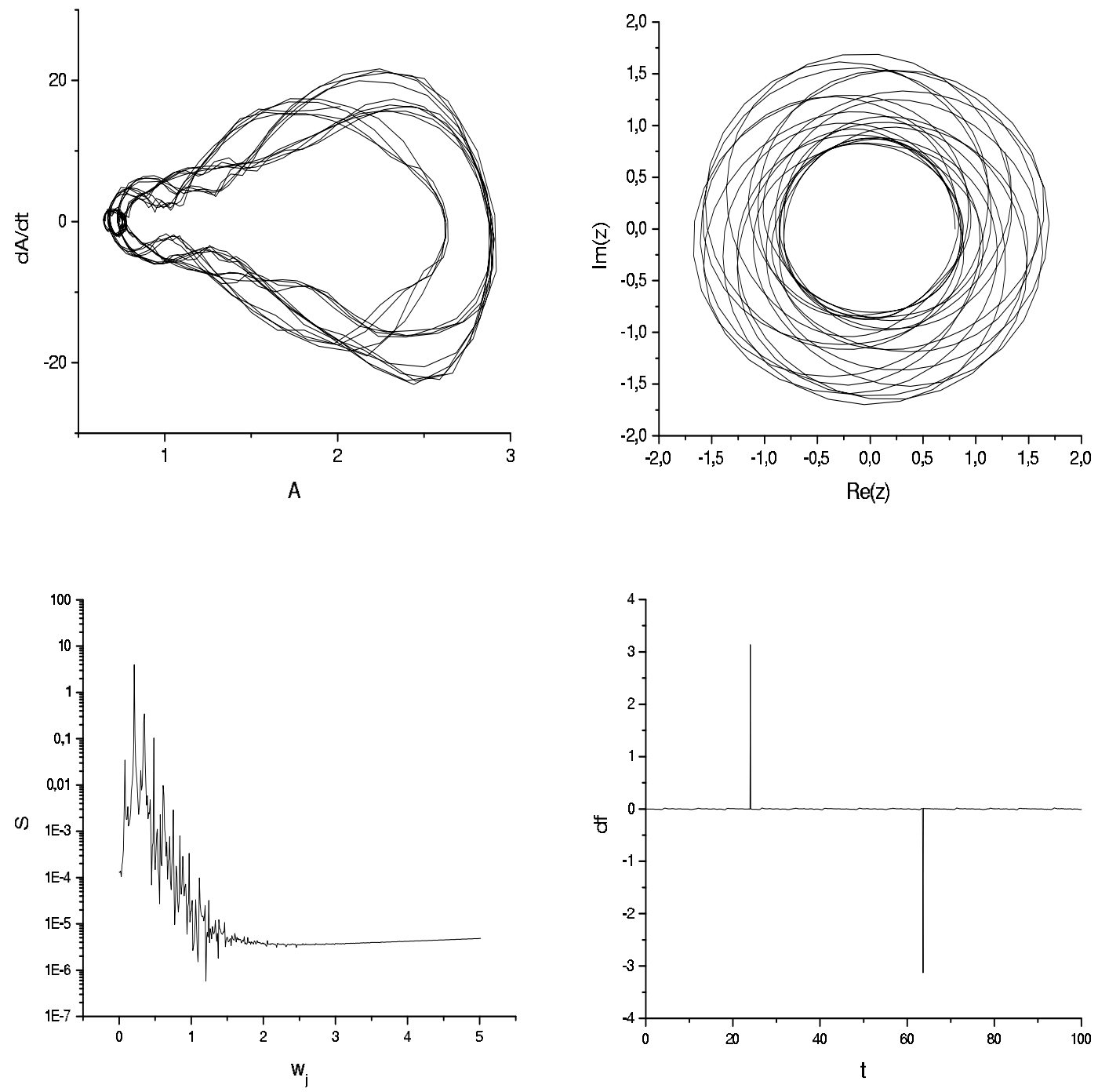

Figure 3: Time evolution of the central oscillator. The values of parameters are $\alpha=1.11$, $J / J_{0}=0.7$ and $M=14.92$. The initial condition is given by Eq. (34). 

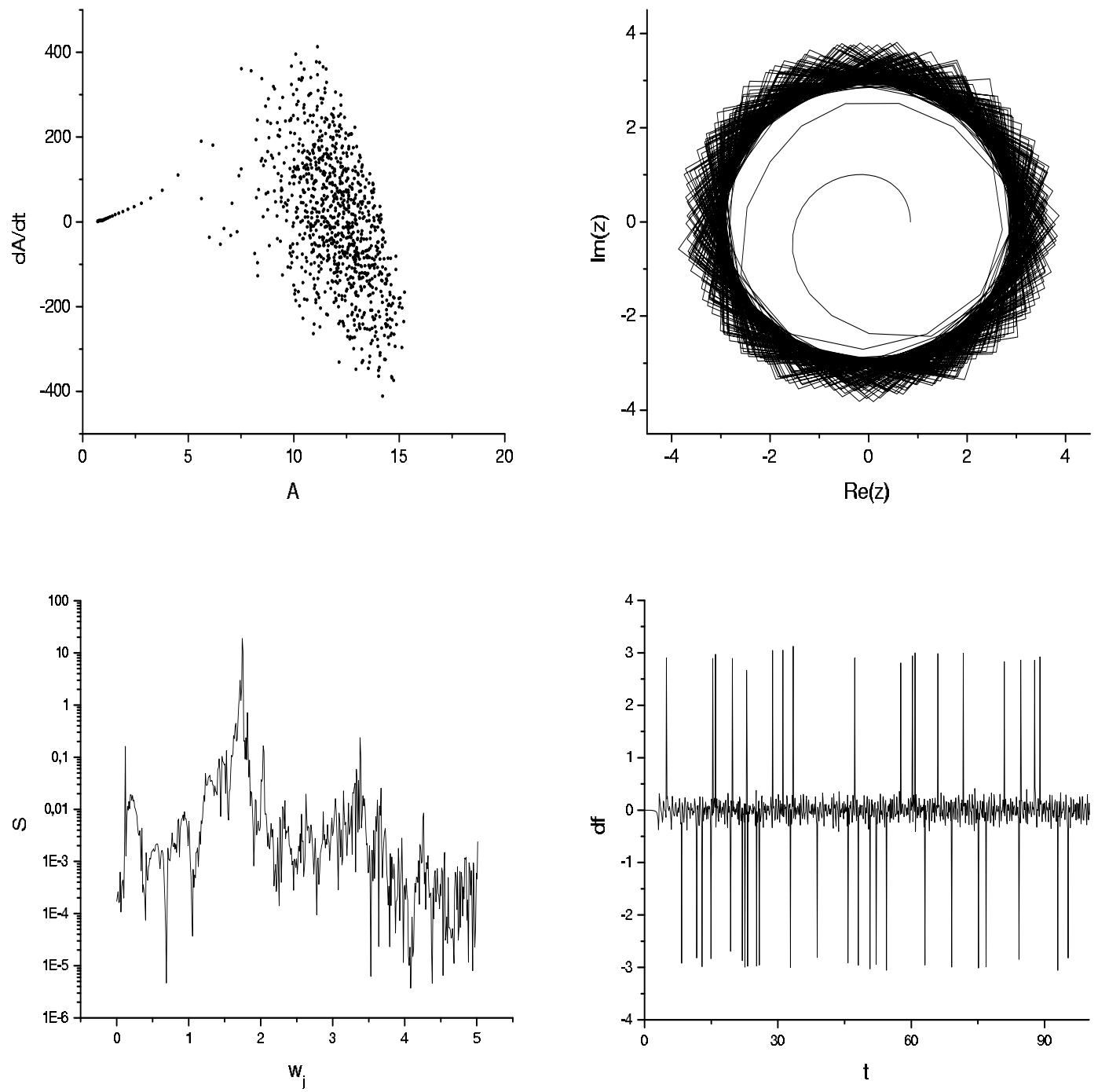

Figure 4: Time evolution of the central oscillator. The values of parameters are $\alpha=1.11$, $J / J_{0}=0.7$ and $M=16.6$. The initial condition is given by Eq. (34). 

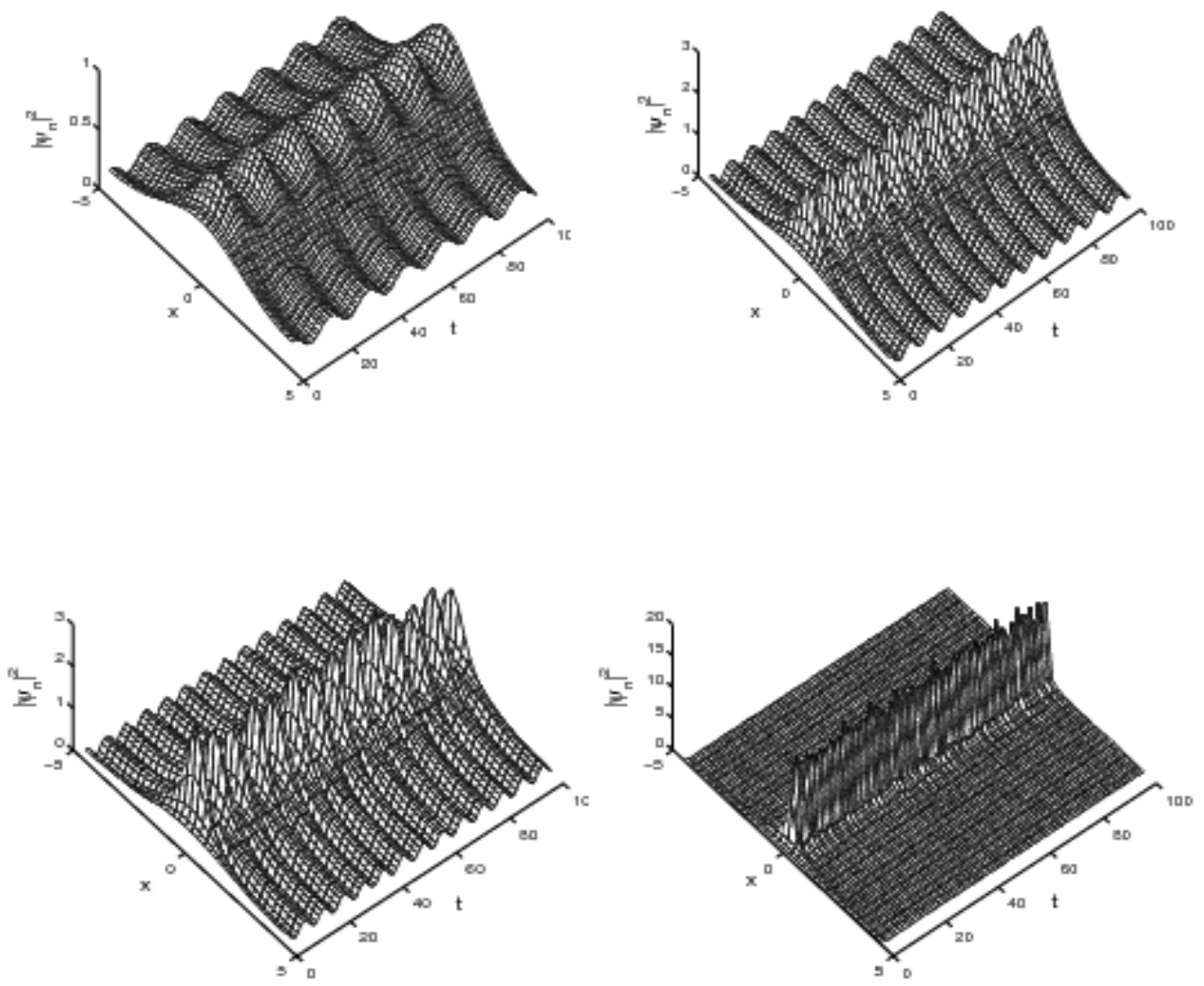

Figure 5: Surfaces $\left|\psi_{n}(t)\right|^{2}$ for the same values of parameters as in Figs. 1-4: $\alpha=1.11$, $J / J_{0}=0.7$ and $M=12.5$ (top left), $M=14.28$ (top right), $M=14.92$ (bottom left) and $M=16.6$ (bottom right). 
Our main goal is to compare solutions of Eq. (25) for different values of $\alpha \in(1,2)$ and consider transition to chaotic dynamics of the chain of oscillators as a function of $\alpha$. The larger is $M$, the stronger is nonlinearity. The larger is $\alpha$, the smaller is LRI. The main differences in the physical properties of the oscillators dynamics are defined by a competition between $\alpha$ and $M$. In all simulations we fix $J / J_{0}=0.7$ as the value close to the one considered in [32. All plots will show the following properties of the oscillators: plane $(d A / d t, A)$ shows projection of the trajectory of the central oscillator in phase space (see definition in (29)); plane (Im $z, \operatorname{Re} z$ ) shows projection of the complex amplitude $z=\psi(0, t)$ of the central oscillator as a function of time; phase difference with the adjacent oscillator to the central one (see Eq. (30)); spectrum of time oscillations of $\psi(0, t)$ (see definition in Eq. (33) ); and surfaces $\left|\psi_{n}(t)\right|^{2}$ vs $t$ and $n$.

First four Figures 1-4 aim to show different regimes of the chain of oscillators behavior for $\alpha=1.11$. For small values of $M$ solutions are quasi-periodic in time with only few modes in the Fourier spectrum. This behaviour for $M=12.5$ is shown on Fig. 1 . The plane $(d A / d t, A)$, the plane (Im $z, R e z)$ and the phase difference plane demonstrate quasi-periodic behavior. As we increase the value of $M$, the spectrum is broadening. Figure 2 demonstrates the behaviour of the system with $\alpha=1.1, J / J_{0}=0.7$ and $M=14.28$. The quasi-periodic structure of the plane $(d A / d t, A)$, the plane (Im $z, R e z)$ and the phase difference plane get more complex. More and more Fourier modes appear in the spectrum. This is even more pronounced for the case $M=14.92$ depicted in Fig. 3, which can be considered as a begining of chaos. The phase difference of two nearby oscillators shows two 'flips' to $\pi$ and $-\pi$ which indicates phase decoherence and transition to chaos. In Fig. 4 for $M=16.6$ the phase difference of two nearby oscillators has many 'flips' to $\pi$ and $-\pi$ and the Fourier spectrum of $\psi(0, t)$ becomes broad what is typical for chaotic dynamics. Surfaces $|\psi(x, t)|^{2}$ for the cases of Figs. 1-4 are shown in Fig. 5. In addition to the chaotization of the solution which was described above, it is seen that with increasing values of $M$, surfaces become more localized around $x=0$, oscillations in the wings of the solutions gradually decrease and the aplitude of the solution is increased. This can be explained by the growth of the nonlinear term with the growth of $M$. The role of a nonlinear coupling becomes more important than coherent connection of oscillators due to the LRI.

Increasing of $\alpha$ leads to appearence of chaotic dynamics for smaller $M$, without significant changes of the diagrams and planes shown in Figs. 1-5. This is demonstrated for $\alpha=1.91$ in Fig. 6. For $\alpha=1.51$ chaos starts at approximately $M=14.28$ and for $\alpha=1.91$ at $M=12.5$. Dynamics for $\alpha<1$ is approximately similar to the dynamics for $1<\alpha<2$ and transition to chaos for $\alpha=0.73$ occurs at $M \sim 18$. The growth of $\alpha$ leads to the increasing of the energy of oscillations in the tails of the solutions. Transition to the strongly developed chaos is not too sharp in time.

All these results could be compared to the standard DNLS equation with the nearestneighbor interaction. Solution of this equation with the symmetric initial condition Eq. (34) is shown in Fig. 7 for $M=12.5$. There are several main distinctions of this case from the transition to chaos when $\alpha<2$ : (i) the symmetry of the solution breaks down for larger $M$ and sharply in time; (ii) the spatial chaos occurs visually earlier than in the case of $\alpha<2$, and this indicates the role of LRI comparing to the standard case of the nearest-neighbor interaction; (iii) another important difference of the onset of chaos in $\alpha$ DNLS and in DNLS can be deduced from the (Im $z, R e z)$ plots; in Fig. 7 (top right panel) for DNLS, trajectory fills space more-or-less uniformly what is typical for Hamiltonian chaos, while in Figs. 3, 4 for $\alpha$ DNLS trajectories looks like in the case of stochastic attractors what is more natural for $\alpha<2$ [56]. There exists an inner part of the diagram that is avoided by the trajectories, at least for the observed time. 

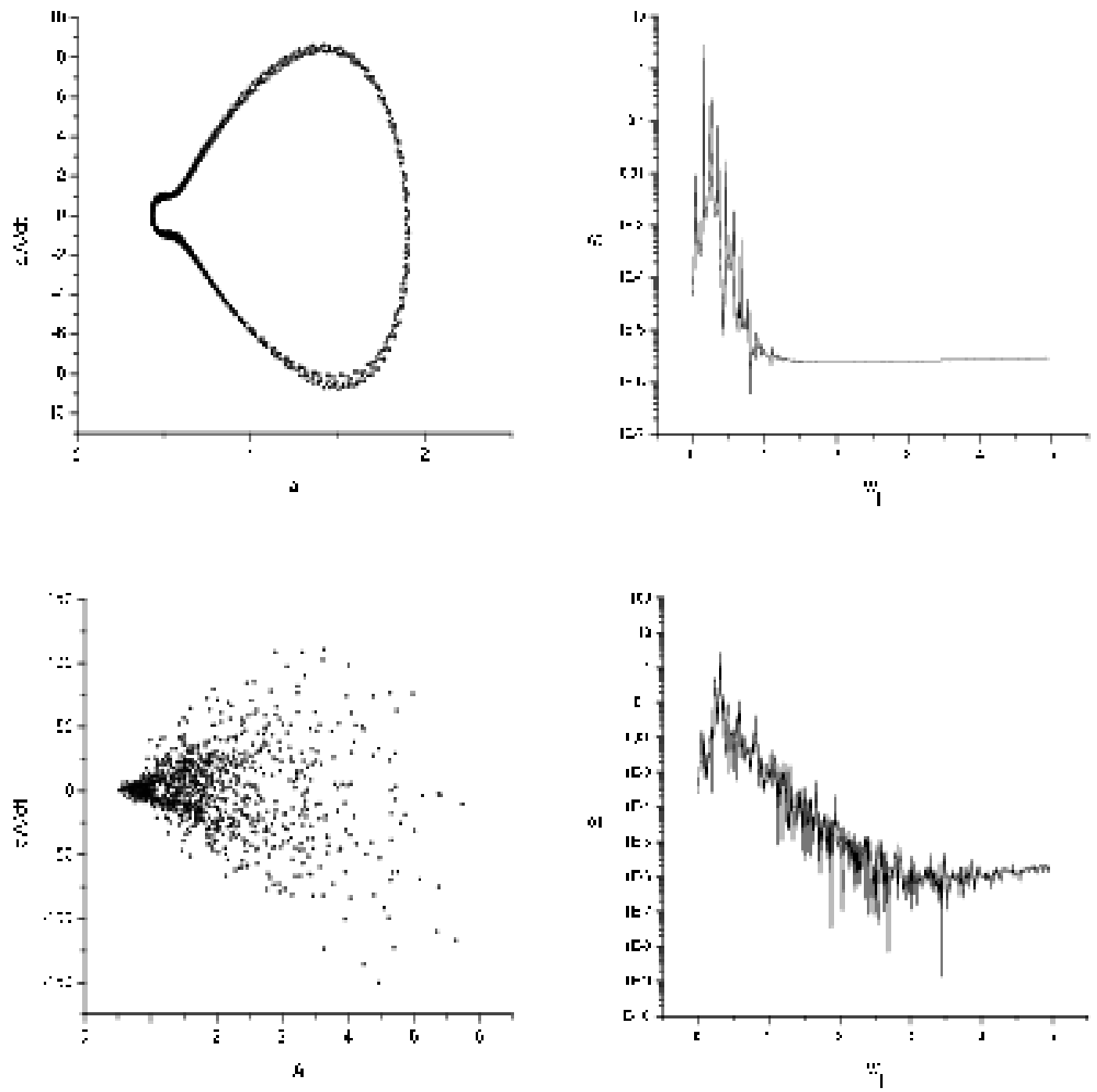

Figure 6: The phase plane of $\psi(0, t)$ and the power spectrum $S$ of $\psi(0, t)$ for parameters $\alpha=1.91, J / J_{0}=0.7, M=10$ (top row) and $M=12.5$ (bottom row). 

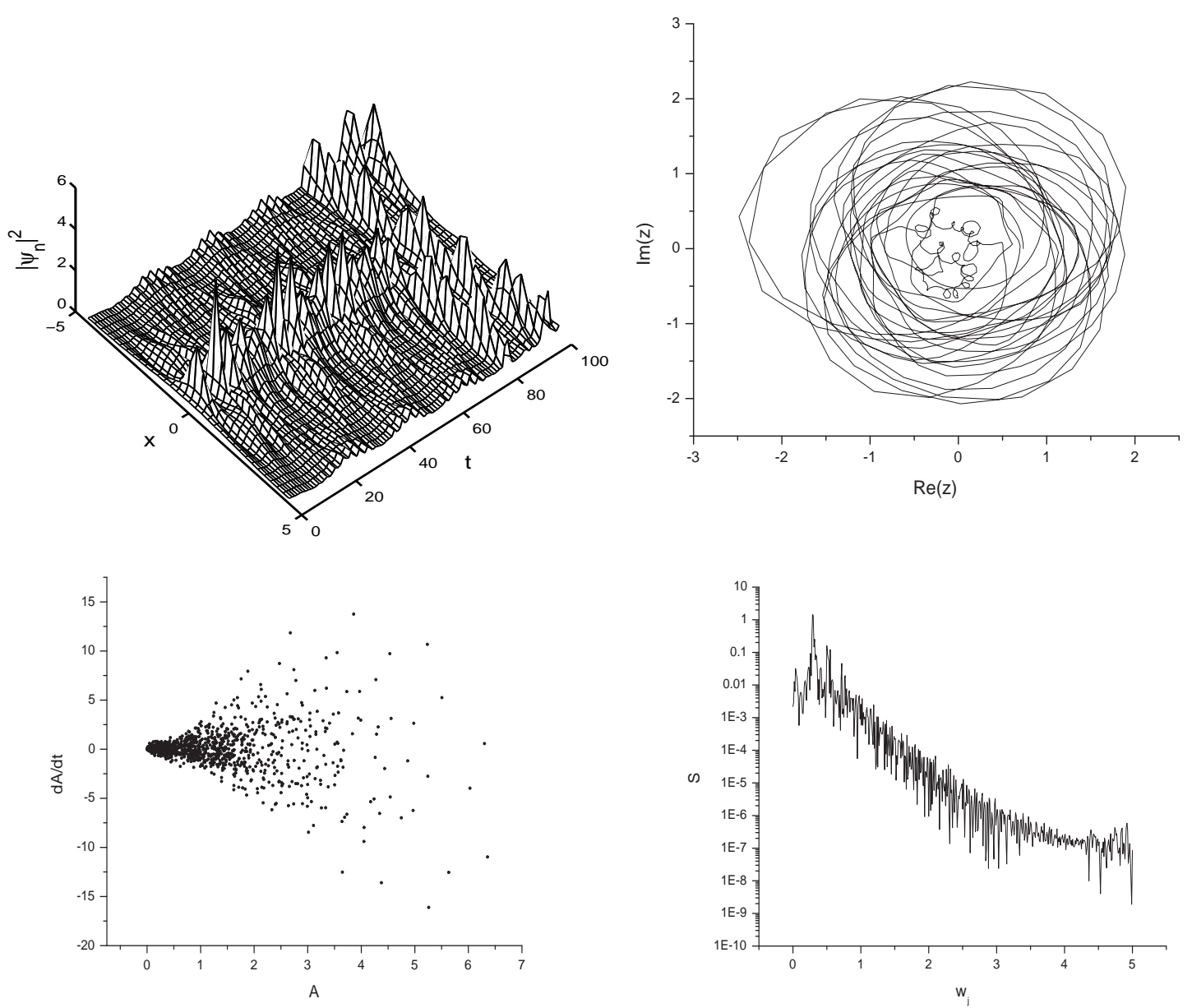

Figure 7: Solution of the standard DNLS equation with $M=12.5$ and $J / J_{0}=0.7$. The initial condition given by Eq. (34). 

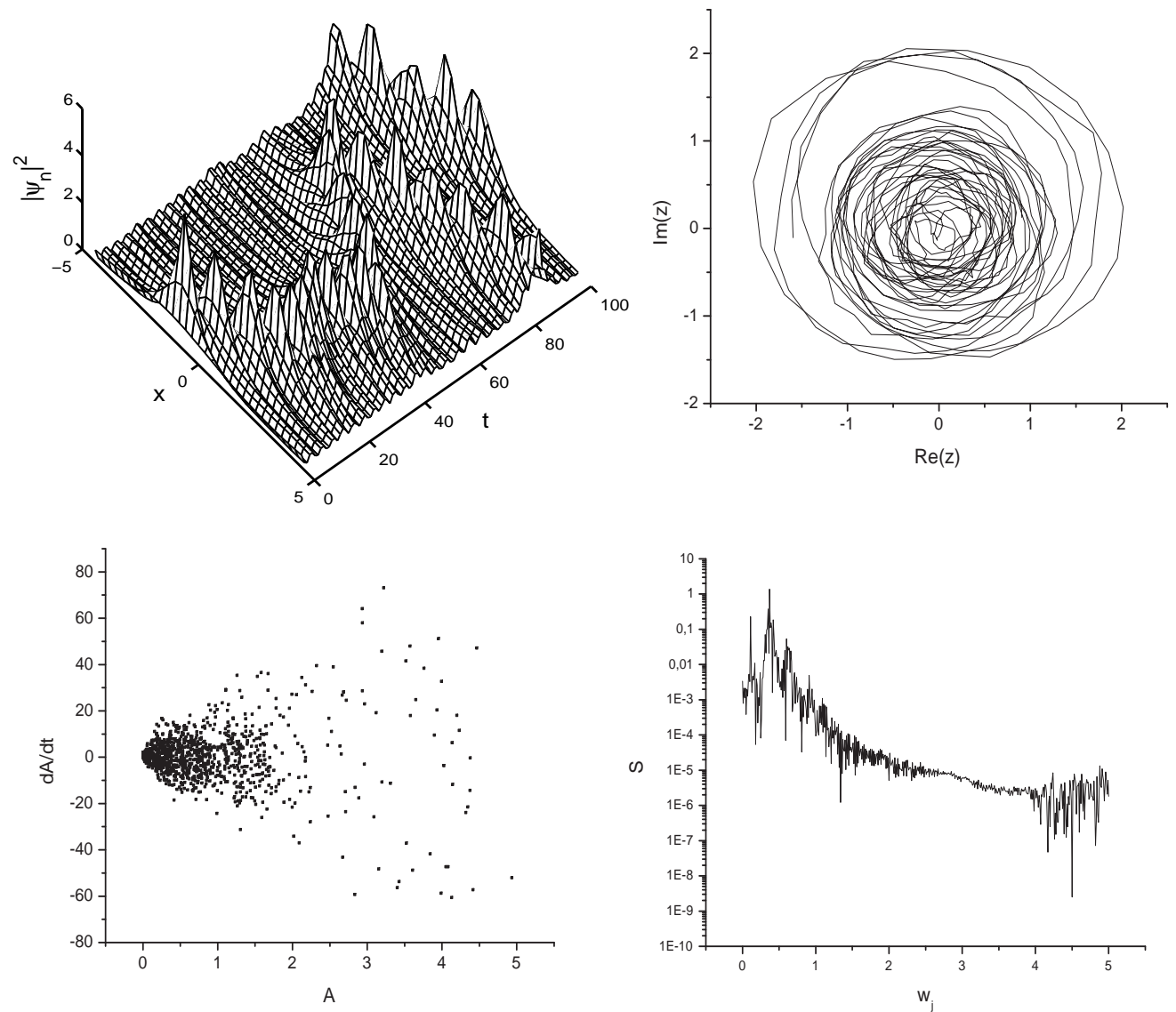

Figure 8: Time evolution of the system of coupled oscillators with LRI and asymmetric initial conditions. The values of parameters are $\alpha=1.11, M=22.22, J / J_{0}=1$. The initial condition is given by Eq. (35). 
The last considered case is the asymmetric initial condition given by Eq. (35). Numerical solution of the equations of motion reveals a difference in this case compared to the symmetric initial condition case. For some values of the excitation number $M$ the numerical solution starts to move in the left or right dirrection. This dirrection can also change randomly in time. Figure 8 shows an example of such behavior for $\alpha=1.11, J / J_{0}=1$ and $M=22.22$. Note, that the power spectrum in this case is also broad.

\section{Conclusion}

One of the main feature of the considered $\alpha$ DNLS model is implementation of a new, additional to the standard DNLS, parameter $\alpha$ that in the continuous limit implies the fractional dynamics described by the FNLS. From another point of view, $\alpha$ is responsible for stong correlations between distant oscillators, i.e. a long-range interaction is introduced through the parameter $\alpha$. This feature of the $\alpha$ DNLS brings a new physics with a new control parameter. The role of the LRI was known before for collective phenomena in complex medium such as chemical or biological set of objects [57, phase transition in one-dimentional systems [58, synchronization 23], regularization in quantum field theory [4]. Our detailed analysis helps to understand some specific properties of destabilization and onset of chaos in $\alpha$ DNLS with $\alpha<2$. Similar analysis can be performed for other models with LRI. An important part of our analysis is utilization of the possibility to transfrom the behavior of discrete chain of interaction objects into the continuous medium equation with the fractional derivatives. This formal procedure raise the question of the discrete-continuous equivalence up to a new level where the appearance of an additional parameter $\alpha$ increases the difficulty the answer the equation.

\section{Acknowledgments}

The authors are grateful to V.E. Tarasov for valuable comments and for the reading of the manuscript. This work was supported by the Office of Naval Research, Grant No. N00014-021-0056 and the NSF Grant No. DMS-0417800.

\section{References}

[1] Y.S. Kivshar, D.E. Pelinovsky, Self-focusing and transverse instabilities of solitary waves, Phys. Rep. 331 (2000), 117-195.

[2] S. Flach, C.R. Willis, Discrete breathers, Phys. Rep. 295, (1998) 181-264.

[3] D. Henning, G.P. Tsironis, Wave transmission in nonlinear lattices, Phys. Rep. 307, (1999) 333-432.

[4] P.G. Kevrekidis, K.O. Rasmussen, A.R. Bishop, The discrete nonlinear Schrodinger equation: A survey of recent results, Int. J. Mod. Phys. B 15 (2001) 2833-2900.

[5] J.C. Eilbeck, M. Johansson, The Discrete Nonlinear Schrödinger equation - 20 Years on, Proc. of the 3rd Conf. Localization \& Energy Transfer in Nonlinear Systems, ed L Vázquez et al (World Scientific, New Jersey, 2003), pp. 44-67.

[6] O.M. Braun, Y.S. Kivshar, Nonlinear dynamics of the Frenkel-Kontorova model. Phys. Rep. 306, (1998) 2-108.

[7] D. Cai, D.W. McLaughlin, J. Shatah, Spatiotemporal chaos and effective stochastic dynamics for a near-integrable nonlinear system, Phys. Lett. A 253, (1999) 280-286. 
[8] D. Cai, D.W. McLaughlin, Chaotic and turbulent behavior of unstable one-dimensional nonlinear dispersive waves, J. Math. Phys. 41, (2000) 4125-4153.

[9] D. Cai, D.W. McLaughlin, J. Shatah, Spatiotemporal chaos in spatially extended systems, Math. Comput. Simulat. 55, (2001) 329-340.

[10] B.M. Herbst, M.J. Ablowitz, Numerically induced chaos in the Nonlinear Schrodinger equation, Phys. Rev. Lett. 62, (1989) 2065-2068.

[11] M.J. Ablowitz, B.M. Herbst, On homoclinic structure and numerically induced chaos for the Nonlinear Schrodinger equation, SIAM J. Appl. Math. 50, (1990) 339-351.

[12] M.J. Ablowitz, C.M. Schober, B.M. Herbst, Numerical chaos, roundoff errors, and homoclinic manifolds, Phys. Rev. Lett. 71, (1993) 2683-2686.

[13] M.J. Ablowitz, B.M. Herbst, C.M. Schober, Computational chaos in the nonlinear Schrödinger equation without homoclinic crossings, Physica A 228, (1996) 212-235.

[14] M.J. Ablowitz, B.M. Herbst, C.M. Schober, Discretizations, integrable systems and computation, J. Phys. A: Math. Gen. 34, (2001) 10671-10693.

[15] D.W. McLaughlin, C.M. Schober, Chaotic and homoclinic behavior for numerical discretizations of the nonlinear Schrödinger equation, Physica D 57, (1992) 447-465.

[16] A. Calini, N.M. Ercolani, D.W. McLaughlin, C.M. Schober, Mel'nikov analysis of numerically induced chaos in the nonlinear Schrödinger equation, Phys. D 89, (1996) 227-260.

[17] G.M. Zaslavsky, Physics of Chaos in Hamiltonian Dynamics, Imperial College Press, 1998 (London).

[18] G.A. Baker, Jr., One-dimensional order-disorder model which approaches a second-order phase transition, Phys. Rev. 122, (1961) 1477-1484.

[19] M. Kac, E. Helfand, Study of several lattice systems with long-range forces, J. Math. Phys. 4, (1963) 1078-1088.

[20] Y. Ishimori, Solitons in a one-dimensional Lennard-Jones lattice, Prog. Theor. Phys. 68 (1982) 402-410.

[21] A.J. Majda, D.W. McLaughlin, E.G. Tabak, A one-dimensional model for dispersive wave turbulence, J. Nonlinear Sci. 6, (1997) 9-44.

[22] D. Cai, A.J. Majda, D.W. McLaughlin, E.G. Tabak, Dispersive wave turbulence in one dimension, Phys. D 152153, (2001) 551-572.

[23] V.E. Tarasov, G.M. Zaslavsky, Fractional dynamics of coupled oscillators with long-range interaction, Chaos 16, (2006) 023110.

[24] J.A. Sepulchre, A. Babloyanz, Controlling chaos in a network of oscillators, Phys. Rev. E 48, (1993) 945-950.

[25] S. Flach, Breathers on lattices with long range interaction, Phys. Rev. E. 58, (1998) R4116R4119.

[26] V.L. Pokrovsky, A. Virosztek, Long-range interactions in commensurate-incommensurate phase-transition, J. Phys. C 16, (1983) 4513-4525.

[27] G. Alfimov, T. Pierantozzi, L. Vazquez, in: A. Le Mehautè, J.A. Tenreiro Machado, L.C. Trigeassou, J. Sabatier (Eds.), Fractional differentiation and its applications, Proceedings of the IFAC-FDA'04 Workshop, Bordeaux, France, July 2004; pp. 153-162.

[28] D.W. McLaughlin, C.M. Schober, Homoclinic manifolds and numerical chaos in the nonlinear Schrödinger equation, Math. Comp. Siml. 37, (1994) 249-264.

[29] B.M. Herbst, M.J. Ablowitz, Numerical Chaos, Symplectic Integrators, and Exponentially Small Splitting Distances, J. Comp. Phys. 105, (1993) 122-132.

[30] B.M. Herbst, F. Varadi, M.J. Ablowitz, Symplectic methods for the nonlinear Schrödinger equation, Math. Comp. Siml. 37, (1994) 353-369. 
[31] M.J. Ablowitz, B.M. Herbst, C.M. Schober, The nonlinear Schrödinger equation: Asymmetric perturbations, traveling waves and chaotic structures, Math. and Computers in Simulation 43, (1997) 3-12.

[32] Y.B. Gaididei, S.F. Mingaleev, P.L. Christiansen, and K.Ø. Rasmussen, Effects of nonlocal dispersive interactions on self-trapping excitations, Phys. Rev. E 55(5), (1997) 6141-6150.

[33] M. Johansson, Y.B. Gaididei, P.L. Christiansen, and K.Ø. Rasmussen, Switching between bistable states in a discrete nonlinear model with long-range dispersion, Phys. Rev. E 57(4), (1998) 4739-4742.

[34] K.Ø. Rasmussen, P.L. Christiansen, M. Johansson, Y.B. Gaididei, S.F. Mingaleev, Localized excitations in discrete nonlinear Schrödinger systems: Effects of nonlocal dispersive interactions and noise, Physica D 113 (1998) 134-151.

[35] P.L. Christiansen, Y.B. Gaididei, F.G. Mertens, S.F. Mingaleev, Multi-component structure of nonlinear exitations in systems with lenth-scale competition, Eur. Phys. J. B 19, (2001) 545-553.

[36] Y.B. Gaididei, S.F. Mingaleev, P.L. Christiansen, and K.Ø. Rasmussen, Effects of nonlocal dispersion on self-trapping excitations, Phys. Lett. A 1996 152-156.

[37] H. Weitzner, G.M. Zaslavsky, Some applications of fractional derivatives, Commun. Nonlin. Sci. Numer. Simul. 8, (2003) 273-281.

[38] A.V. Milovanov, J.J. Rasmussen, Fractional generalization of the Ginzburg-Landau equation: an unconvential approach to critical phenomena in complex media, Phys. Lett. A 337, (2005) 75-80.

[39] N. Laskin, G.M. Zaslavsky, Nonlinear chain dynamics with long-range interaction, Physica A 368, (2006) 38-54.

[40] V.E. Tarasov, G.M. Zaslavsky, Fractional Ginzburg-Landau equation for fractal media, Physica A 354, (2005) 249-261.

[41] V.E. Tarasov, Psi-series solution of fractional Ginzburg-Landau equation, J. Phys. A. 39, (2006) 8395-8407.

[42] S.G. Samko, A.A. Kilbas, O.I. Marichev, Fractional Integrals and Derivatives Theory and Applications, Gordon and Breach, New York, 1993.

[43] N. Korabel, G.M. Zaslavsky, V.E. Tarasov, Coupled oscillators with power-law interaction and their fractional dynamics analogues, in press, Commun. in Nonlin. Sci. and Comput. Simulations, (2006).

[44] R.P.A. Lima, M.L. Lyra, J.C. Cressoni, Multifractality of one electron eigen states in 1D disordered long range models, Physica A 295, (2001) 154-157.

[45] D.W. Schaefer, J.E. Martin, P. Wiltzius, et al., Fractal geometry of colloidal aggregates, Phys. Rev. Lett. 52, (1984) 2371-2374.

[46] P. Pfeifer, D. Avnir, Chemistry in noninteger dimensions between two and three. I. Fractal theory of heterogeneous surfaces, J. Chem. Phys. 79, 3558-3565.

[47] E. Goldfain, Derivation of the fine structure constant using fractional dynamics, Chaos, Solitons and Fractals 17 (2003) 811-818.

[48] E. Goldfain, Renormalization group and the emergence of random fractal topology in quantum field theory, Chaos, Solitons and Fractals 19 (2004) 1023-1030.

[49] E. Goldfain, Complexity in quantum field theory and physics beyond the standard model, Chaos, Solitons and Fractals 28 (2006) 913-922.

[50] A. Erdèlyi, W. Magnus, F. Oberhettinger, F.G. Tricomi, Higher Transcendental Functions, Vol. 1, New York, Krieger, (1981) pp. 30-31. 
[51] L. Lewin, Polylogarithms and associated functions, New York: North-Holland; 1981.

[52] K.B. Oldham, J. Spanier, The Fractional Calculus, Academic Press, New York, 1974.

[53] K.S. Miller, B. Ross, An Introduction to the Fractional Calculus and Fractional Differential Equations, Wiley, New York, 1993.

[54] I. Podlubny, Fractional Differential Equations, Academic Press, New York, 1999.

[55] F. Mainardi, R. Gorenflo, Feller fractional diffusion and Levy stable motions, in: O.E. Barndorff-Nielsen, S.E. Graversen, T. Mikosch (Eds.), Proceedings of the International Conference on "Levy Processes: Theory and Applications", MPS-misc. 1999-11, pp. 11122 ,

[56] G.M. Zaslavsky, A.A. Stanislavsky, M. Edelman, Chaotic and pseudochaotic attractors of perturbed fractional oscillator, CHAOS 16 (2006), 0131021-0131026.

[57] Y. Kuramoto, Self-entrainment of a population of coupled non-linear oscillators. in International Symposium on Mathematical Problems in Theoretical Physics. Ed. H. Araki (Springer, Berlin, 1975) pp.420-422.

[58] F.J. Dyson, Existence of a phase-transition in a one-dimensional Ising ferromagnet. Commun. Math. Phys. 12 (1969) 91-107; Non-existence of spontaneous magnetization in a onedimensional Ising ferromagnet. Commun. Math. Phys. 12 (1969) 212-215; An Ising ferromagnet with discontinuous long-range order. Commun. Math. Phys. 21 (1971) 269-283. 